\title{
Is Consciousness a Nonspatial Phenomenon?
}

\section{Ståle Gundersen}

\begin{abstract}
Colin McGinn has argued that consciousness is a nonspatial phenomenon. McGinn's arguments for the nonspatiality of consciousness are presented and then criticized. It is concluded that consciousness may be as spatial as electric charge and different kinds of abilities.
\end{abstract}

Key words: Descartes, Colin McGinn, consciousness, nonspatial phenomenon

A ccording to Descartes, the physical world (res extensa) is essentially extended, whereas mental substances (res cogitans) are nonspatial entities; i.e., they are indivisible and have no shape, size, texture, and cannot be located in space. Colin McGinn agrees with Descartes that the mind is nonspatial, but McGinn's perspective on the mind-body problem is a kind of property dualism, not substance dualism, entailing that conscious states are unexplainable and nonspatial properties of brains. ${ }^{1}$

In the first part of the paper I will present McGinn's arguments for the nonspatiality of consciousness. In the second part I will cast doubt about these arguments and argue that consciousness may be as spatial as electric charge and abilities.

\section{McGinn's Arguments}

According to McGinn, a phenomenon is nonspatial if it does not fall under the pre-theoretical or scientific spatial predicates like 'place', 'area', 'volume', 'texture', 'configuration', 'solidity', 'shape' and 'being composed of spatial parts'. The scientific spatial predicates are derived from scientific theories about space, including Quantum Mechanics and the Special and General Theories of Relativity. ${ }^{2}$ McGinn makes two different arguments for

1 See Colin McGinn, "Consciousness and space," in Journal of Consciousness Studies, 2 (1995), 220-230; Colin McGinn, The Character of Mind. Second Edition (Oxford: Oxford University Press, 1998); Colin McGinn, The Mysterious Flame (New York: Basic Books, 1999).

${ }^{2}$ Unless dealing specifically with the distinction between pre-theoretical and scientific spatial predicates, I will simply write 'spatial predicates.'

(C) 2011 Ståle Gundersen

http://www.kritike.org/journal/issue 9/gundersen june2011.pdf ISSN 1908-7330 
the nonspatiality of consciousness: 'the predicate argument' and 'the observability argument.'

The predicate argument. The essence of the predicate argument is that, because we cannot apply all the spatial predicates to describe conscious states, conscious states must be nonspatial. Consider a visual experience $(\mathrm{E})-\mathrm{a}$ yellow flash. Associated with $\mathrm{E}$ is a neural event $(\mathrm{N})$ in the brain. McGinn claims that $\mathrm{N}$ does admit of spatial description, but not $\mathrm{E}$. $\mathrm{E}$ is not located at any specific place, it takes up no particular volume of space, and it has no spatial dimensionality, texture or shape. Asking for E's spatial properties is a category mistake, like asking for the spatial properties of numbers. ${ }^{3}$ Conscious states do not fall under spatial predicates; one does not speak of a twinge of anxiety as occurring five centimeters behind the right ear, or assert that an experience of smell of Camembert is $5 \mathrm{~cm}$ left of the visual experience of red. Thus, we cannot use spatial predicates to describe the relations between different experiences, and since conscious states do not fall under spatial predicates they must be nonspatial.

The observability argument. The root of McGinn's observability argument is the premise that conscious states cannot be perceived: "consciousness enables us to perceive the world, but it is not itself a perceptible thing." "4 Even though an organism's behavior and neuronal activity is perceptible (by using brain-imaging technologies like PET and fMRI), we cannot perceive its experiences. According to McGinn, consciousness is not unobservable in the same way as elementary particles, because elementary particles are definitely located in space, and the main cause of their unobservability is simply their smallness. McGinn claims that consciousness is nonspatial in a more fundamental way, because even God could not perceive conscious states and abstract entities as spatial phenomena, but could perceive elementary particles, if they exist, as distributed in space. ${ }^{5}$

McGinn criticizes a popular argument for spatial conscious states, that we in our daily lives locate conscious states in the vicinity of the brain or the body. McGinn agrees that most people do not believe that a person's conscious states are located away from one's body, and so one does locate conscious states spatially, at least in this rudimentary sense. However, McGinn does not think that this common sense conception of conscious states undermines the thesis of the nonspatiality of the mental, because we do not locate conscious states by perceiving them to be close to bodies and brains, but only by trading upon certain causal considerations.

I am where that body is whose physical states beat most directly on my mental state; and my states of consciousness are situated in the vicinity of that brain whose activity is most directly implicated in the causal relations controlling my mental life. ${ }^{6}$

\footnotetext{
${ }^{3}$ McGinn, "Consciousness and space," 220.

${ }^{4}$ McGinn, The Mysterious Flame, 113.

${ }^{5}$ McGinn, "Consciousness and space," 221.

${ }^{6}$ Ibid., 221-222.
} 
Thus, minds have only a derivative relation to space by being correlated with brain states that can be spatially located. McGinn asserts that, even though consciousness has a spatial-physical foundation, consciousness is not necessarily spatial. Our knowledge of physical reality, our knowledge about spatial-physical properties and entities, is not sufficient to explain consciousness. Therefore, there must be some hidden nonspatial features that contribute to determining consciousness.

The unobservability or unperceivability of consciousness constitutes an important premise in McGinn's argument for consciousness' nonspatiality. Consciousness is not perceptible, and no type of sense organs or measuring instruments could be imagined that would enable us to perceive consciousness. ${ }^{7}$ Consciousness is therefore a nonspatial phenomenon.

1. Conscious states are phenomena that cannot be observed.

2. A phenomenon is spatial if and only if it can be observed.

\section{Conscious states are nonspatial phenomena}

McGinn's first premise is that conscious states cannot be observed. Consciousness is in this sense 'invisible', we cannot directly observe other organisms' experiences. This premise is closely related to the problem of other minds, because it states that observations cannot decide what kind of experiences the organisms have, or whether they are conscious at all. Observations cannot tell us whether snails, spiders and other insects have conscious experiences. Let's say that according to theory T1, organism $\mathrm{O}$ is conscious but according to theory $\mathrm{T} 2, \mathrm{O}$ is non-conscious. These conclusions may be based on some observable characteristics like the amount of neurons. T1 and T2 are not testable in the sense that we can decide by observation which theory is correct, because as McGinn argues, consciousness is unobservable.

The second premise is a biconditional containing two different statements. First, if a phenomenon can be observed, it must be spatial. The truth of this conditional seems obvious, because it is difficult to imagine observing something nonspatial. But this conditional is irrelevant for the subsequent arguments. The second premise also asserts that, if a phenomenon is spatial, it can in principle be observed. This looks prima facie plausible because even spatial entities like bacteria, despite being invisible to the naked eye, are detectable by advanced instruments like electron microscopes. Elementary particles, if not observable to the naked eye or even by instruments, are at least observable in principle. Consciousness, as a genuine nonspatial phenomenon, cannot be observed even in principle, and therefore constitutes a nonspatial dimension in the universe in addition to the known spatial dimensions. We

${ }^{7}$ McGinn, The Mysterious Flame, 114. 
have no conception what it is to perceive conscious states as spatial entities, consciousness is therefore nonspatial simpliciter. ${ }^{8}$

\section{Some problems with McGinn's arguments}

The observability argument. The second premise in McGinn's argument - the conditional that states that a phenomenon is spatial only if it can be observed - is problematic because we are not entitled to pass from the statement that a phenomenon is 'invisible' or unobservable to the conclusion that it is nonspatial. This is an invalid inference, because not being perceptible does not entail nonspatiality, as illustrated by Quantum Mechanics. Quantum Mechanics teaches that we can only know the probability that a single particle is inside or outside a region of space. According to the most common interpretation, Quantum Mechanics supplies complete information regarding the particle's pre-measurement state, and in its pre-measurement state the particle is without a definite position. This pre-measurement state is called a 'superposition' and implies that the particle is both inside and outside the region at the same time. Even though we cannot observe a particle in its superpositioned state, the particle in this state is nonetheless a spatial phenomenon, because it is described by the Schrödinger equation (the wave-function), which refers to spatial coordinates. The moment we measure the particle's position, the wave-function will collapse and then the particle will have a definite position. Since a particle's super-positioned state is a spatial phenomenon that cannot be observed, the second premise is thrown into question. Unobservability and nonspatiality are not co-extensional.

The predicate argument. McGinn often refers to our use of pretheoretical spatial predicates and common sense conception of conscious states - e.g. that pains have no shape, solidity and size etc - as an argument for the nonspatiality of consciousness. It sounds strange to talk about beliefs, desires and pains as if they have volumes, solidity and lengths. However, common sense is not a criterion for truth. Neuropsychological data indicate that mental activity is the same as brain activity, so mental states may be usefully described using spatial concepts. But McGinn takes a very conservative attitude towards how scientists should be allowed to talk about mental states. However, Jerome Shaffer asserts that language rules for locating mental states in space (the brain) can be adopted because nothing in the way we teach the use of mental predicates rules out spatially located mental states. ${ }^{9}$ Due to brain-imaging technologies (PET and fMRI), among other factors, it has become almost

8 There has been a debate whether McGinn's thesis of the nonspatiality of consciousness is consistent with the Special Theory of Relativity. According to the Special Theory of Relativity, if something is located in time, then it must also be located in space. Since conscious states are located in time, they must also be located in space. See Sophie R. Allen, "A space oddity: Colin McGinn on consciousness and space," in Journal of Consciousness Studies, 13 (2006), 61-82. I don't intend to contribute to this debate in this paper.

9 Jerome Shaffer, "Could mental states be brain processes?" in The Journal of Philosophy, 58 (1961), 813-822. 
commonplace to refer to thoughts and other mental states as 'located in the brain.' One may therefore contend that we have already adapted Shaffer's language rules.

On the other hand, this critique may be overly simplistic. We are not introspectively aware that pain has a volume, shape, texture or solidity, and this is a phenomenological fact that must be considered. A problem with this reply, however, is that it is a fallacy to pass from ' $\mathrm{I}$ am not introspectively aware that conscious states are spatial' to 'I am introspectively aware that conscious states are nonspatial.' Conscious states may have properties (like being spatial) that are not revealed by introspection. ${ }^{10}$ This restates the well-worn argument that introspection represents a limited source of knowledge about our mental states. In fact, McGinn agrees that introspection is a limited source of knowledge about the nature of consciousness and how consciousness is related to the physical world, but he does not seem to realize that this may undermine his own conclusion of the nonspatiality of consciousness. ${ }^{11}$

If we agree with McGinn that conscious states do not satisfy the criteria of being spatial phenomena, we may also be forced to deny that abilities are spatial phenomena. The ability to go skiing is a property of certain persons' bodies. This property is a disposition, and a person has this ability also when the ability is not manifested. The ability to go skiing may be difficult to locate (is it located in the brain, in certain parts of the body, or throughout the whole body?), and it seems even more difficult, not to say meaningless or impossible, to ascribe a size, texture, solidity or shape to abilities. Is my ability to go skiing behind, to the left or under my ability to play ping-pong? Do these abilities have the same shape or texture? We do not speak about abilities as if they are spatial in this sense, but it is nevertheless quite obvious that abilities are spatial phenomena. Abilities are probably easier to explain and describe than conscious states, but the fact that a phenomenon (like consciousness) is difficult or impossible to explain or describe does not imply that it is nonspatial.

A possible objection to this argument is that some features of complex abilities like skiing or ping-pong may be nonspatial in the sense that it is impossible for a system to have some of these abilities without also having some nonspatial conscious states. It is, however, difficult to find a convincing argument for a necessary relationship between the mentioned abilities and conscious states. Also, it is easy to imagine a simple robotic system, which we have no reason to believe is conscious, but which has certain abilities like moving around and discriminating between different kinds of objects. We may further suppose that these abilities are well integrated with each other and

${ }^{10}$ For an analogous argument see David M. Armstrong, "The headless woman illusion and the defence of materialism," in Analysis, 29 (1968), 48-49. Antoine Arnauld used the same kind of argument when he criticized Descartes' substance dualism, for even though thought is the mind's essential property, it doesn't follow that the mind may not have other essential properties also (e.g. being physical) not revealed by introspection. Arnauld's argument is found in the fourth set of objections to the Meditations.

11 See Colin McGinn, The Problem of Consciousness (Cambridge: Blackwell, 1991). 


\section{6}

NONSPATIAL PHENOMENON

distributed all over the robot's body and brain. We can then repeat mutatis mutandis the argument above to show that abilities constitute counterexamples to McGinn's criteria of spatiality. ${ }^{12}$

Another similarity between abilities and conscious states is that we can use some (but not all) spatial predicates to describe and refer to them. From a neuro-psychological point of view it is neither false nor meaningless to say that the causes of mathematical abilities are mainly located in the brain, but the ability to go skiing is more distributed. Mathematical abilities and skiing are manifested by the whole person, but they may nevertheless be located more precisely according to their causal grounding. Analogously, conscious states like anxiety and experiences of colors seem to be related to different parts of the brain: anxiety is related to the amygdala and visual experiences are related to the visual cortex. Neuroscientists use spatial concepts in this loose sense when they describe conscious states. This may be considered sufficient to conclude that conscious states are spatial. ${ }^{13}$ However, McGinn may be reluctant to accept this conclusion, as he appears to think that a phenomenon is spatial if and only if it can be subsumed under all spatial predicates. He says that,

... to allow that consciousness can be roughly located is not to grant it the full panoply of spatial predications. We still do not get predications of shape, size, dimensionality and so on. And this shows that such spatiality as we do allow to mental matters is of a second-class and derivative nature. ${ }^{14}$

First, it is not clear what is meant by 'second class and derivative spatiality', and it is not clear how this kind of spatiality is distinguished from real or 'first class' spatiality. Spatiality does not seem to be a phenomenon that permits of degrees, because either a phenomenon is spatial or it is not. Second, it is difficult to accept that it must be a necessary condition for a phenomenon $\mathrm{x}$ to be spatial that $\mathrm{x}$ must fall under all spatial predicates like size, texture, solidity etc. Given this criterion of spatiality, even abilities must be considered nonspatial, because they do not have size, texture etc. Even the electrons' charge will turn out to be nonspatial according to this criterion, because it is utterly meaningless to measure the diameter or volume of a particular charge. The property charge, which is measured in Coulomb, is not measured in volume

12 It is important to notice that I have only argued that not all abilities must have conscious aspects, but this is not an argument against David Lewis' contention that conscious states are abilities. See David Lewis, "What experience teaches," in The Nature of Consciousness. Philosophical Debates, ed. by Ned Block, Owen Flanagan and Güven Güzeldere (Cambridge Mass.: MIT Press, 1998).

13 It is debated within the cognitive sciences how widely distributed conscious states are in the brain, but even if conscious states have no precisely defined boundaries this fact in itself does not constitute an argument against their spatiality, since clouds too lack precise spatial boundaries but are obviously spatial phenomena.

14 McGinn, "Consciousness and space," 222. 
units, but the entities that have a particular charge may have a volume, or at least be located in space. ${ }^{15}$ Thus, that we can use a proper subset of spatial predicates (e.g. that it can be located) to describe $\mathrm{x}$ seems to be a sufficient condition for phenomenon $\mathrm{x}$ to be spatial. From the semantic fact that it is not reasonable or meaningful to use all spatial predicates to describe $\mathrm{x}$, we need not conclude that $\mathrm{x}$ is nonspatial, for in that case charge and abilities would also be nonspatial phenomena. Consciousness may therefore be a spatial property of the body in the same way as abilities and charge are properties of certain entities. Consciousness, abilities and charge are not measured in volume units, but they may still be spatial phenomena in virtue of being properties of physical entities located in space. McGinn has claimed that,

In order to solve the mind-body problem we need, at a minimum, a new conception of space...That which we refer to when we use the word 'space' has a nature that is quite different from how we standardly conceive it to be; so different, indeed, that it is capable of 'containing' the nonspatial (as we now conceive it) phenomenon of consciousness. ${ }^{16}$

However, a new conception of space is not necessary in order to solve the mind-body problem, because consciousness is as spatial as ability and charge. This simplifies McGinn's account of the mind-body problem, but in itself does not give us any reason to be sanguine about the prospect of solving the mind-body problem. We are still left with the hard problem of how to explain the emergence of consciousness (qualia) from brain processes. Even though consciousness is analogous with ability, and abilities can in principle be given an explanation based on the body's physiological constitution, the problem of the emergence of consciousness is still a mystery. However, theories of space appear to be orthogonal to theories about consciousness and the mind-body problem.

Center of Philosophy, University of Stavanger, Norway

\section{References}

Allen, S.R., "A space oddity: Colin McGinn on consciousness and space," in Journal of Consciousness Studies, 13 (2006), 61-82.

Armstrong, D.M., "The headless woman illusion and the defence of materialism," in Analysis, 29 (1968), 48-49.

\footnotetext{
15 McGinn of course agrees that electric charge is a spatial feature since charge is a physical property of entities that can be located in space.

16 McGinn, "Consciousness and space," 226.
} 


\section{NONSPATIAL PHENOMENON}

Lewis, D.K., "What experience teaches," in The Nature of Consciousness.

Philosophical Debates, ed. by Block, N., Flanagan, O. and Güzeldere, G.

(Cambridge Mass.: MIT Press, 1998).

McGinn, C., The Problem of Consciousness (Cambridge: Blackwell, 1991).

McGinn, C., "Consciousness and space," in Journal of Consciousness Studies, 2 (1995), 220-230.

McGinn, C., The Character of Mind, second edition, (Oxford: Oxford University Press, 1998).

McGinn, C., The Mysterious Flame (New York: Basic Books, 1999).

Shaffer, J., "Could mental states be brain processes?" in The Journal of Philosophy, 58 (1961), 813-822. 